\title{
On the mathematical controllability in a simple growth tumors model by the internal localized action of inhibitors
}

\author{
J.I. Díaz ${ }^{1,2}$ and J.I. Tello ${ }^{1}$
}

\begin{abstract}
We study a model of growth of tumors with a free boundary, delaying the tumor region. We take into account the presence of inhibitors and its interaction with the nutrients. We study the approximate controllability of the internal distribution of density of cells, that is proportional to concentration of nutrients, injecting inhibitor in a small inner region $\omega_{0}$.
\end{abstract}

\section{The model}

In this paper we study the controllability of the growth of tumors by the internal localized action of inhibitors on a simplified mathematical model. The tumor, formed by life cells, is assumed to have a density proportional to the concentrations of a nutrient $\hat{\sigma}(x, t), x=\left(x_{1}, x_{2}, x_{3}\right)$, mainly oxygen or glucose. We study the behavior of the tumor after angiogenesis, the formation of capillary sprouts from blood vessels, in response to externally supplied chemical stimuli (see, e.g. Chaplain and Anderson [1996]). Once the angiogenesis occurs, the tumor receives nutrient from the vessels (process named vasculature). We assume that the tumor occupates a radially symmetric ball of $\mathbb{R}^{3}$ of radius $R(t)$, which is unknown (reason why is usually denoted as the free boundary of the problem). Denoting by $\sigma_{B}$ the constant nutrient concentration in the vasculature, $\hat{r}_{1}$ the rate, per unit length, of nutrient transferred to the tissue, $\hat{\sigma}$ satisfies the equation

$$
\frac{\partial \hat{\sigma}}{\partial t}-d_{1} \Delta \hat{\sigma}-\hat{r}_{1}\left(\sigma_{B}-\hat{\sigma}\right)+\lambda_{1} \hat{\sigma}+\lambda \hat{\beta}=0, \quad|x|<R(t), t \in(0, T) .
$$

Here $d_{1}$ is the diffusion coefficient of the nutrient concentration and $\lambda_{1} \hat{\sigma}, \lambda \hat{\beta}$ represent the consume rate of nutrient and inhibitor, respectively.

The density of the inhibitor $\hat{\beta}(x, t)$ is assumed to satisfy a similar reaction - diffusion equation,

$$
\frac{\partial \hat{\beta}}{\partial t}-d_{2} \Delta \widehat{\beta}-\widehat{r}_{2}\left(\beta_{B}-\hat{\beta}\right)+\lambda_{2} \widehat{\beta}=f \chi_{\omega_{0}}, \quad|x|<R(t), t \in(0, T),
$$

\footnotetext{
${ }^{1}$ Departamento de Matemática Aplicada, Universidad Complutense de Madrid, 28040 Madrid

${ }^{2}$ Partially supported by the DGES (Spain) project REN2000/0766
} 
with $d_{2}$ the diffusion coefficient, $\beta_{B}$ the critical value of the inhibitor concentration for vasculature, $\hat{r}_{2}$ the rate, per unit length, of inhibitor transferred to the tissue, and $\lambda_{2} \beta$ is the inhibitor consumption rate. The permanent supply of inhibitors is assumed to be localized on a small domain $\omega_{0}$ with a rate given by $f$ (the control of the problem).

According the mass conservation principle, assuming the cell mass density constant, the tumor mass is proportional to the volume $\frac{4}{3} \pi R(t)^{3}$. The balance between birth and death cells is determinate by the concentration of nutrient and inhibitor. Denoting by $\widehat{S}$ the above balance, after normalizing we obtain the law

$$
\frac{d}{d t}\left(\frac{4}{3} \pi R^{3}(t)\right)=\int_{\{|x|<R(t)\}} \widehat{S}(\widehat{\sigma}(x, t), \widehat{\beta}(x, t)) d x, \quad x \in \mathbb{R}^{3} .
$$

According the inhibitor nature and the tumor tissue, the function $\hat{S}$ has different representations. In any case we shall assume trough the paper that, $\widehat{S} \in W^{1, \infty}\left(\mathbb{R}^{2}\right)$.

For the sake of notation we shall assume that the diffusion coefficients are given by a unique positive constants, $d_{1}=d_{2}=d$. Thus by normalizing the unknown densities

$$
\sigma:=\hat{\sigma}-\frac{\hat{r}_{1} \sigma_{B}\left(\hat{r}_{2}+\lambda_{2}\right)+\lambda \hat{r}_{2} \beta_{B}}{\left(\hat{r}_{1}+\lambda_{1}\right)\left(\hat{r}_{2}+\lambda_{2}\right)}, \quad \beta:=\hat{\beta}-\frac{\hat{r}_{2} \beta_{B}}{\hat{r}_{2}+\lambda_{2}}
$$

and denoting by

$$
r_{1}:=\widehat{r}_{1}+\lambda_{1}, \quad r_{2}:=\widehat{r}_{2}+\lambda_{2}, \quad S(\sigma, \beta):=\frac{3}{4 \pi} \widehat{S}(\widehat{\sigma}, \widehat{\beta}),
$$

we arrive to the concrete formulation of the mathematical model under consideration

$$
\begin{gathered}
\frac{\partial \sigma}{\partial t}-d \Delta \sigma+r_{1} \sigma+\lambda \beta=0, \quad|x|<R(t), t \in(0, T), \\
\frac{\partial \beta}{\partial t}-d \Delta \beta+r_{2} \beta=f \chi_{\omega_{0}}, \quad|x|<R(t), t \in(0, T), \\
R(t)^{2} \frac{d R(t)}{d t}=\int_{|x|<R(t)} S(\sigma, \beta) d x, q q u a d R(0)=R_{0}, t \in(0, T), \\
\sigma(x, 0)=\sigma_{0}(x), \quad \beta(x, 0)=\beta_{0}(x), \quad|x|<R_{0}, \\
\sigma(x, t)=\overline{\bar{\sigma}}, \beta(x, t)=\overline{\bar{\beta}}, \quad|x|=R(t), t \in(0, T),
\end{gathered}
$$

where $R_{0}>0$, the normalized nutrient and inhibitor densities at the exterior of the tumor $\overline{\bar{\sigma}}, \overline{\bar{\beta}}$, the initial densities $\left(\sigma_{0}, \beta_{0}\right)$ are assumed to be given. We shall assume that $\left(\sigma_{0}, \beta_{0}\right) \in W^{2, \infty}\left(B\left(R_{0}\right)\right)$. The mathematical treatment of this model has a long history, (see See Greenspan [1972], Adams [1986], Byrne - Chaplain [1996], Byrne [1999], Cui - Friedman [1999], Reitich - Friedman [1999], Díaz - Tello [1999]). A recent reference containing details on the notion of weak solution and existence and uniqueness is the authors work (Díaz - Tello [2000]). The main results of this paper shows that this type of action by the inhibitor allows to control (in the usual weak sense typical of parabolic system) the tumor density. This is formulated in the following terms: 
Theorem 1.1 Given $T>0, \omega_{0} \subset B\left(R_{0} \exp \left\{-\|S\|_{L^{\infty}} T\right\}\right), \epsilon>0$, and $\hat{\sigma}^{d} \in L_{l o c}^{p}\left(\mathbb{R}^{3}\right)$, for some $p>1$, there exists $f \in L^{p}\left((0, T) \times \omega_{0}\right)$ such that, if $(\sigma, \beta, R)$ is the solution of the problem (1.1)-(1.5), then

$$
\left\|\sigma(T)-\sigma^{d}\right\|_{L^{p}(B(R(T)))} \leq \epsilon
$$

where $\sigma^{d}:=\hat{\sigma}^{d} \chi_{B(R(T))}$.

Due to some technical reasons, we shall prove the theorem firstly for $p \geq 5$, (necessary in the proof of Lemma 2.1) and then for all $p>1$.

We shall prove the result in several steps. For $n \in \mathbb{N}$, we start by assuming $R_{n}(t)$ prescribed and look for a control $f_{n}$ in $\omega_{0}$ such that the solution $\left(\sigma_{n}, \beta_{n}\right)$ of problem (1.1)-(1.5), satisfies (1.6). Then we obtain $R_{n+1}$ and $f_{n+1}$ from $\left(\sigma_{n}, \beta_{n}\right)$ which allow to find $\left(\sigma_{n+1}, \beta_{n+1}\right)$. The proof of the theorem uses some methods introduced in the study of the approximate controllability (name attributed to conclusions as (1.6)) by different authors (see Lions [1990], [1991], Puel - Fabre - Zuazua [1995], and Díaz - Ramos [1995]). In spite of the large literature on this type of methods, very few seems to be known for the case of systems (see also Díaz-Ramos [1998] for a higher order equation). Some numerical experiences could be developed in the line of the works Glowinski-Lions [1995] and Diaz-Ramos [2000]. Iterating the process we obtain a sequence $\left(R_{n}, f_{n}, \sigma_{n}, \beta_{n}\right)$, we show that there exists a subsequence such that converges to the searched control $f$ and the associate solution of problem (1.1)-(1.5).

\section{Regularity and uniqueness of problem (1.1)-(1.5)}

Although the existence of weak solutions of problem (1.1)-(1.5), was established by previous authors, (see Díaz - Tello [2000]), we shall need some extra information which is collected in this section.

In order to prove the regularity of the solutions we use the change of variables and unknowns, introduced in Díaz - Tello [2000],

$$
\begin{gathered}
\tilde{x}:=\left(\tilde{x}_{1}, \tilde{x}_{2}, \tilde{x}_{3}\right)=\frac{x}{R(t)}, \quad \tilde{t}(t):=\int_{0}^{t} R^{-2}(\rho) d \rho, \\
u(\tilde{x}, \tilde{t}):=\sigma(R(t(\tilde{t})) \tilde{x}, t(\tilde{t}))-\overline{\bar{\sigma}}, \quad v(\tilde{x}, \tilde{t}):=\beta(R(t(\tilde{t})) \tilde{x}, t(\tilde{t}))-\overline{\bar{\beta}} .
\end{gathered}
$$

(Notice since $R$ is a continuous function and $\frac{1}{R^{2}(t)}>0$, we obtain that $\tilde{t}(t) \in C^{1}([0, \tilde{T}])$, and by the Theorem of Implicit Function, there exists the inverse function, $t(\tilde{t}) \in$ $C^{1}([0, T])$.

Let $B=\left\{\tilde{x} \in \mathbb{R}^{3}, \quad|\tilde{x}|<1\right\}$. Problem (1.1)-(1.5) can be equivalently formulated as

$$
\begin{array}{cl}
\frac{\partial u}{\partial \tilde{t}}-d \Delta u-R^{2} \dot{R} \tilde{x} \cdot \nabla u+R^{2} r_{1} u=R^{2}\left(r_{1} \overline{\bar{\sigma}}+\lambda(v+\overline{\bar{\beta}})\right), & \tilde{x} \in B, \tilde{t} \in(0, \tilde{T}), \\
\frac{\partial v}{\partial \tilde{t}}-d \Delta v-R^{2} \dot{R} \tilde{x} \cdot \nabla v+R^{2} r_{2} v=R^{2} f \chi_{\tilde{\omega}_{0}}-R^{2} r_{2} \overline{\bar{\beta}}, \quad \tilde{x} \in B, \tilde{t} \in(0, \tilde{T}),
\end{array}
$$




$$
\begin{gathered}
R(\tilde{t}) \frac{d}{d \tilde{t}} R(\tilde{t})=\int_{B} S(u(\tilde{x}, \tilde{t})+\overline{\bar{\sigma}}, v(\tilde{x}, \tilde{t})+\overline{\bar{\beta}}) d \tilde{x}, \quad R(0)=R_{0}, \\
u(\tilde{x}, \tilde{t})=v(\tilde{x}, \tilde{t})=0, \quad \tilde{x} \in \partial B, \tilde{t} \in(0, \tilde{T}), \\
u(\tilde{x}, 0)=u_{0}(\tilde{x})=\sigma_{0}\left(\tilde{x} R_{0}\right), \quad v(\tilde{x}, 0)=v_{0}(\tilde{x})=\beta_{0}\left(\tilde{x} R_{0}\right),
\end{gathered}
$$

where $\tilde{T}=\tilde{t}(T)$ and $\tilde{\omega}_{0}^{\tilde{t}}=\left\{\tilde{x} \in B\right.$ such that $\left.R(t(\tilde{t})) \tilde{x} \in \omega_{0}\right\}$, for any $\tilde{t} \in[0, \tilde{T}]$.

Lemma 2.1 Under the assumptions of Theorem 1.1, for $p \geq 5$, the solution $(u, v, R)$ of problem (2.3)-(2.7), satisfies

$$
u \in L^{q}\left(0, \tilde{T}: W^{2, q}(B)\right) \cap W^{1, q}\left(0, \tilde{T}: L^{q}(B)\right),
$$

for all $1<q<\infty$ and

$$
v \in L^{p}\left(0, \tilde{T}: W^{2, p}(B)\right) \cap W^{1, p}\left(0, \tilde{T}: L^{p}(B)\right) .
$$

Proof. By Theorem 1 of Díaz and Tello [2000] we know that

$$
(u, v, R) \in\left[L^{2}\left(0, \tilde{T}: H^{1}(B)\right)\right]^{2} \times W^{1, \infty}(0, \tilde{T}) .
$$

Then the linear parabolic operator

$$
\mathcal{L} v:=\frac{\partial v}{\partial \tilde{t}}-d \Delta v-R^{2} R^{\prime} \tilde{x} \cdot \nabla v+R^{2} r_{2} v
$$

admits a fundamental solution (see Friedman [1964]) and, since $v_{0} \in H^{2}(B), f \in$ $L^{p}((0, T) \times B)$, we get

$$
\left.v \in W^{1, p}((0, \tilde{T}) \times B) \cap L^{p}\left(0, \tilde{T}: W^{2, p}(B)\right)\right),
$$

(see e.g. Ladyzenkaya, Solonnikov and Uralceva [1991], Theorem 9.1, Chap IV). Since $p>4, W^{1, p}((0, T) \times B) \subset L^{\infty}([0, \tilde{T}] \times B)$, and then

$$
\left.u \in W^{1, q}((0, T) \times B) \cap L^{q}\left(0, T: W^{2, q}(B)\right)\right),
$$

for $q \leq \infty$. Consequently, we obtain $R(t) \in W^{2, p}(0, T)$.

As a consequence of the lemma we obtain,

Corollary 2.1 By using that $W_{0}^{1, p}(B \times[0, \tilde{T}]) \subset L^{\infty}(B \times[0, \tilde{T}])$, if $p>4$, then $u, v \in$ $L^{\infty}(B \times[0, \tilde{T}])$.

On the other hand, the continuous embeddings

$$
\begin{aligned}
& \left.W^{1, q}((0, T) \times B) \cap L^{q}\left(0, T: W^{2, q}(B)\right)\right) \subset L^{2}\left(0, T: W^{1, \infty}(B)\right), \\
& \left.W^{1, p}((0, \tilde{T}) \times B) \cap L^{p}\left(0, \tilde{T}: W^{2, p}(B)\right)\right) \subset L^{2}\left(0, T: W^{1, \infty}(B)\right),
\end{aligned}
$$

and the reciprocal change of variables and unknown (2.1), (2.2), leads to 
Corollary 2.2 Under the assumptions of Theorem 1.1, we have

$$
\int_{0}^{T}\|\sigma\|_{W^{1, \infty}(R(t))}^{2}+\|\beta\|_{W^{1, \infty}(R(t))}^{2} d t \leq k_{0} .
$$

The uniqueness of solutions is proved in the next proposition.

Proposition 2.1 Let $f \in L^{p}\left(\omega_{0} \times(0, T)\right)$ with $p \geq 5$, and $\left(\sigma_{0}-\overline{\bar{\sigma}}, \beta_{0}-\overline{\bar{\beta}}\right) \in W^{2, s}\left(B\left(R_{0}\right)\right) \cap$ $H_{0}^{1}\left(B\left(R_{0}\right)\right)$, for $s>4$. Then, there exists a unique solution of the problem (1.1)-(1.5).

Proof. We shall show that if we assume that there exist two different solutions, $\left(\sigma_{1}, \beta_{1}, R_{1}\right)$ and $\left(\sigma_{2}, \beta_{2}, R_{2}\right)$, we get a contradiction. Let

$$
R(t)=\min \left\{R_{1}(t), R_{2}(t)\right\}, \quad \sigma=\sigma_{1}-\sigma_{2}, \quad \beta=\beta_{1}-\beta_{2} .
$$

Then $(\sigma, \beta, R)$ satisfies the problem,

$$
\begin{array}{cc}
\frac{\partial \sigma}{\partial t}-d \Delta \sigma+r_{1} \sigma+\lambda \beta=0, & |x|<R(t), t \in(0, T), \\
\frac{\partial \beta}{\partial t}-d \Delta \beta+r_{2} \beta=0, \quad & |x|<R(t), t \in(0, T), \\
\sigma(x, 0)=0, \quad \beta(x, 0)=0, \quad|x|<R_{0}, \\
\sigma(x, t)=\sigma_{1}(x, t)-\sigma_{2}(x, t), & |x|=R(t), t \in(0, T), \\
\beta(x, t)=\beta_{1}(x, t)-\beta_{2}(x, t), & |x|=R(t), t \in(0, T) .
\end{array}
$$

We introduce a new unknown defined by

$$
z=k_{1} \sigma-k_{2} \beta
$$

with

$$
\begin{array}{llll}
k_{1}=1, & k_{2}=\frac{\lambda}{r_{1}-r_{2}}, & \text { if } & r_{1} \neq r_{2}, \\
k_{1}=\frac{1}{2}, & k_{2}=\frac{\lambda}{r_{1}-2 r_{2}}, & \text { if } & r_{1}=r_{2} \neq 0,
\end{array}
$$

and by $z=e^{-\lambda t} \sigma-\beta$ if $r_{1}=r_{2}=0$. By construction we have

$$
\begin{cases}\frac{\partial z}{\partial t}-d \Delta z+r_{1} z=0, & |x|<R(t), t \in(0, T), \\ z(x, 0)=0, & |x|<R_{0}, \\ z(x, t)=k_{1} \sigma(x, t)-k_{2} \beta(x, t), & |x|=R(t), t \in(0, T) .\end{cases}
$$

Now we prove a preliminary result:

Lemma 2.2 Let $z$ be the solution of problem (2.13) and $\beta$ the solution of problem (2.9), (2.12), then $e^{r_{1} t} z$ and $e^{r_{2} t} \beta$ take their maximum and minimum on $|x|=R(t)$. 
Proof. Multiplying the equation (2.13) by $e^{r_{1} t}$ we obtain that $e^{r_{1} t} z$ satisfies

$$
\begin{cases}\frac{\partial}{\partial t}\left(e^{r_{1} t} z\right)-d \Delta\left(e^{r_{1} t} z\right)=0, & |x|<R(t), t \in(0, T), \\ z(x, 0)=0, & |x|<R_{0}, \\ e^{r_{1} t} z(x, t)=e^{r_{1} t}\left(k_{1} \sigma(x, t)-k_{2} \beta(x, t)\right), & |x|=R(t), t \in(0, T) .\end{cases}
$$

Repeating the operation, we obtain $e^{r_{2} t} \beta$ satisfies the equation,

$$
\begin{cases}\frac{\partial}{\partial t}\left(e^{r_{2} t} \beta\right)-d \Delta\left(e^{r_{2} t} \beta\right)=0, & |x|<R(t), t \in(0, T), \\ \beta(x, 0)=0, & |x|<R_{0}, \\ e^{r_{2} t} \beta(x, t)=e^{r_{2} t}\left(\beta_{1}(x, t)-\beta_{2}(x, t)\right), & |x|=R(t), t \in(0, T) .\end{cases}
$$

By Corollary 2.1, we know that

$$
|\sigma(x, t)| \leq K, \quad|\beta(x, t)| \leq K, \text { for any } t \in[0, T], \text { and a.e. } x \in B(R(t)),
$$

and then, $e^{r_{1} t} z$ and $e^{r_{2} t} \beta$ are bounded. Let

$$
\begin{aligned}
& z^{* *}=\max \left\{e^{r_{1} t} z(x, t), t \in[0, T], x \in \partial B(R(t))\right\}, \\
& z_{* *}=\min \left\{e^{r_{1} t} z(x, t), t \in[0, T], x \in \partial B(R(t))\right\}, \\
& \beta^{* *}=\max \left\{e^{r_{2} t} \beta(x, t), t \in[0, T], x \in \partial B(R(t))\right\}, \\
& \beta_{* *}=\min \left\{e^{r_{2} t} \beta(x, t), t \in[0, T], x \in \partial B(R(t))\right\} .
\end{aligned}
$$

Let $T_{k}$ and $T^{k}$ be defined by

$$
T_{k}(s)= \begin{cases}s, & \text { if } s>k \\ k, & \text { if } s, \leq k\end{cases}
$$

and

$$
T^{k}(s)= \begin{cases}k, & \text { if } s, \geq k, \\ s, & \text { if } s,<k .\end{cases}
$$

Taking $T_{0}\left(e^{r_{1} t} z-z^{* *}\right)$ as test function in (2.14), integrating by parts in $B(R(t))$, and by Leibnitz Theorem, after some manipulations, we arrive to

$$
\frac{d}{d t} \int_{B(R(t))}\left[T_{0}\left(e^{r_{1} t} z-z^{* *}\right)\right]^{2} d x \leq 0
$$

and we deduce that $e^{r_{1} t} z$ takes his maximum in $|x|=R(t)$. In the same way, taking $T^{0}\left(e^{r_{1} t} z-z_{* *}\right)$ as test function we obtain

$$
z_{* *} \leq e^{r_{1} t} z \leq z^{* *}
$$

The proof of

$$
\beta_{* *} \leq e^{r_{2} t} \beta \leq \beta^{* *},
$$

is analogous. 
End of the proof of Proposition 2.1. Given $t \in[0, T]$, we can suppose, without lost generality, $R_{1}(t) \leq R_{2}(t)$, otherwise the argument is similar by changing $R_{1}$ for $R_{2}$. Using that

$$
\begin{gathered}
R_{1}^{2}(t) \dot{R}_{1}(t)-R_{2}^{2}(t) \dot{R}_{2}(t)=\int_{B(R(t))}\left(S\left(\sigma_{1}, \beta_{1}\right)-S\left(\sigma_{2}, \beta_{2}\right)\right) d x- \\
\int_{R_{1}(t)<|x|<R_{2}(t)} S\left(\sigma_{2}, \beta_{2}\right) d x .
\end{gathered}
$$

Since $S$ is bounded, then

$$
\left|\int_{R_{1}(t)<|x|<R_{2}(t)} S\left(\sigma_{2}, \beta_{2}\right) d x\right| \leq N\left|R_{1}^{3}(t)-R_{2}^{3}(t)\right| \leq M\left|R_{1}(t)-R_{2}(t)\right|,
$$

where $M$ depends only of $|S|_{L^{\infty}}$. Since $S$ is Lipschitz continuous, integrating in time, it results

$$
\begin{gathered}
\int_{0}^{T} \int_{B(R(t))}\left|S\left(\sigma_{1}, \beta_{1}\right)-S\left(\sigma_{2}, \beta_{2}\right)\right| d x d t \leq \\
\int_{0}^{T} \int_{B(R(t))}|S|_{W^{1, \infty}\left(\mathbb{R}^{2}\right)}(\sup |\sigma|+\sup |\beta|) d x d t \leq \\
\int_{0}^{T} \int_{B(R(t))} k_{0}\left(\frac{1}{k_{1}} \sup \left|z+k_{2} \beta\right|+\sup |\beta|\right) d x d t \leq \\
\int_{0}^{T} \int_{B(R(t))} C(\sup |z|+\sup |\beta|) d x d t \leq \\
\int_{0}^{T} \int_{B(R(t))} C\left(\sup \left|e^{-r_{1} t} e^{r_{1} t} z\right|+\sup \left|e^{-r_{2} t} e^{r_{2} t} \beta\right|\right) d x d t \leq \\
\int_{0}^{T} \int_{B(R(t))} C\left(e^{\left|r_{1}\right| T} \sup \left|e^{r_{1} t} z\right|+e^{\left|r_{2}\right| T} \sup ^{r_{2} t} \beta \mid\right) d x d t \leq \\
\int_{0}^{T} \int_{B(R(t))} k_{3}\left(\sup \left|e^{r_{1} t} z\right|+\sup \left|e^{r_{2} t} \beta\right|\right) d x .
\end{gathered}
$$

From Lemma 2.2, we know

$$
\int_{0}^{T} \int_{B(R(t))} \sup \left|e^{r_{1} t} z(x, t)\right| d x d t \leq e^{r_{1} T} \frac{3 \pi}{4} R^{3}(t) \int_{0}^{T} \sup _{|x|=R(t)}|z(x, t)| d t .
$$

By Corollary 2.2, we deduce that

$$
\int_{0}^{T}\left(\left\|\sigma_{2}\right\|_{W^{1, \infty}(B(R(t))}^{2}+\left\|\beta_{2}\right\|_{W^{1, \infty}(B(R(t))}^{2}\right) d t \leq K_{0},
$$

and consequently,

$$
\int_{0}^{T}\|z\|_{W^{1, \infty}(B(R(t)))}^{2} d t \leq K
$$

Since

$$
e^{r_{1} t} z(x, t)=e^{r_{1} t}\left(k_{1}\left(\sigma_{2}(x, t)-\overline{\bar{\sigma}}\right)-k_{2}\left(\beta_{2}(x, t)-\overline{\bar{\beta}}\right)\right) \text {, on }|x|=R(t),
$$


we deduce

$$
\begin{gathered}
e^{r_{1} T} \frac{3 \pi}{4} R^{3}(t) \int_{0}^{T} \sup _{|x|=R(t)}|z(x, t)| d t \leq \\
k_{4} \int_{0}^{T}\left\|\sigma_{2}\right\|_{W^{1, \infty}\left(B\left(R_{2}(t)\right)\right)}+\left\|\beta_{2}\right\|_{W^{1, \infty}\left(B\left(R_{2}(t)\right)\right)}\left|R_{1}(t)-R_{2}(t)\right| d t \leq \\
k_{4} \sup _{0<t<T}\left|R_{1}(t)-R_{2}(t)\right| T^{\frac{1}{2}} \int_{0}^{T}\left(\left\|\sigma_{2}\right\|_{W^{1, \infty}\left(B\left(R_{2}(t)\right)\right)}^{2}+\left\|\sigma_{2}\right\|_{W^{1, \infty}\left(B\left(R_{2}(t)\right)\right)}^{2}\right) d t \leq \\
k \sup _{0<t<T}\left|R_{1}(t)-R_{2}(t)\right| T^{\frac{1}{2}} .
\end{gathered}
$$

In the same way,

$$
\int_{0}^{t} \int_{B(R(t))} k_{3} \sup |\beta| \leq k \sup _{0<t<T}\left|R_{1}(t)-R_{2}(t)\right| T^{\frac{1}{2}} .
$$

Then

$$
\int_{0}^{t}\left|R_{1}^{2}(t) \dot{R}_{1}(t)-R_{2}^{2}(t) \dot{R}_{2}(t)\right| d t \leq C_{0} \sup _{0<t<T}\left|R_{1}(t)-R_{2}(t)\right|\left(T+T^{\frac{1}{2}}\right) .
$$

Denoting by $\delta=\max _{t \in[0, T]}\left\{R_{1}(t)-R_{2}(t)\right\}$, we obtain

$$
\left|R_{1}^{3}(t)-R_{2}^{3}(t)\right| \leq 3 C_{0} \delta\left(T+T^{\frac{1}{2}}\right),
$$

and since $\left|R_{1}^{3}(t)-R_{2}^{3}(t)\right| \geq 3 R_{0}^{2}\left|R_{1}(t)-R_{2}(t)\right|$, we conclude, $\delta \leq k_{0} \delta\left(T+T^{\frac{1}{2}}\right)$. Then, if $T<T_{1}=\min \left\{\frac{1}{4 k_{0}^{2}}, 1\right\}$, necessarily $R_{1}(t)=R_{2}(t)$. Since $e^{r_{1} t} z$ and $e^{r_{2} t} \beta$ take his maximum and minimum on $R(t)=R_{1}(t)=R_{2}(t)$ and it is zero, then $\beta=0$ and $z=0$, and we deduce $\beta=0$ and $\sigma=0$.

Repeating the same argument, now from $T_{1}$ we conclude the uniqueness of solutions for a $T>0$ arbitrary.

\section{Approximate controllability: Proof of Theorem 1.1}

The next result shows the conclusion of Theorem 1.1 (the so called approximate controllability in $L^{p}$ ) under some particular assumptions (mainly when $R(t)$ is a priori prescribed).

Proposition 3.1 Let $\omega_{0} \subset B\left(R_{0} \exp \left\{-\|S\|_{L^{\infty}} T\right\}\right.$, and $\sigma_{0}=\beta_{0}=\underline{\underline{\sigma}}=\underline{\underline{\beta}}=0$. Let $R \in W^{1, \infty}(0, T)$ a given function such that $R(0)=R_{0},|\dot{R}| \leq\|S\|_{L^{\infty}} R_{0} \exp \left\{\|S\|_{L_{\infty} T} T\right.$. Then, given $\hat{\sigma}^{d} \in L_{\text {loc }}^{2}\left(\mathbb{R}^{3}\right)$, there exists $f \in L^{p}\left(\omega_{0} \times(0, T)\right)$, with $p \geq 5$, such that, if $(\sigma, \beta)$ is the solution of problem (1.1), (1.2), (1.4) and (1.5), with $R(t)$ prescribed, then

$$
\left\|\sigma(T)-\sigma^{d}\right\|_{L^{p}(B(R(T)))} \leq \epsilon
$$

where $\sigma^{d}=\left.\hat{\sigma}^{d}\right|_{B(R(T))}$. 

by

Proof. Let $p^{\prime}=\frac{p}{p-1}$, we consider the functional $J: L^{p^{\prime}}(B(R(T))) \longrightarrow \mathbb{R}$ defined

$$
J\left(\varphi^{0}\right)=\frac{1}{p^{\prime}} \int_{0}^{T} \int_{\omega_{0}}|\psi(x, t)|^{p^{\prime}} d x d t+\epsilon\left\|\varphi^{0}\right\|_{L^{p^{\prime}}(B(R(T)))}-\int_{B(R(T))} \sigma^{d} \varphi^{0} d x
$$

for $\varphi_{0} \in L^{p^{\prime}}(B(R(T)))$, where $\psi$ is the component of the solution $(\varphi, \psi)$ of the "dual" problem

$$
\begin{gathered}
-\frac{\partial \varphi}{\partial t}-d \Delta \varphi-r_{1} \varphi=0, \quad|x|<R(t), t \in(0, T), \\
-\frac{\partial \psi}{\partial t}-d \Delta \psi-r_{2} \psi+\lambda \varphi=0, \quad|x|<R(t), t \in(0, T), \\
\varphi(x, T)=\varphi_{0}(x), \quad \psi(x, T)=0, \quad|x|<R(T), \\
\varphi(x, t)=0, \quad \psi(x, t)=0, \quad|x|=R(t), \quad t \in(0, T) .
\end{gathered}
$$

We point out that the existence of a weak solutions of $(3.1)-(3.4),(\varphi, \psi)$ can be obtained as in section 2, by making the change of variable (2.1), (2.2), (see Tello [2001]).

In order to prove the uniqueness of solutions, we suppose there exists two solutions, $\left(\varphi_{1}, \psi_{1}\right),\left(\varphi_{2}, \psi_{2}\right)$, then $\varphi:=\varphi_{1}-\varphi_{2}$, satisfies the equation (3.1), taking $|\varphi|^{p^{\prime}-2} \varphi$ as test function, and integrating by parts, it results,

$$
-\frac{d}{d t} \int_{B(R(t))}|\varphi|^{p^{\prime}} d x \leq r_{1} \int_{B(R(t))}|\varphi|^{p^{\prime}} d x
$$

by Gronwall's Lemma, since $\varphi(T)=0$, we obtain $\varphi=\varphi_{1}-\varphi_{2}=0$. Once proved $\varphi \equiv 0$, in the same way, $\psi:=\psi_{1}-\psi_{2}$ satisfies (3.2), taking $|\psi|^{p^{\prime}-2} \psi$ as test function, we obtain $\psi \equiv 0$, and consequently, the uniqueness is proved.

Let us assume that $J$ is convex, continuous and coercive (in the sense that $\lim \inf J \rightarrow$ $\infty$ if $\left\|\varphi^{0}\right\|_{L^{p^{\prime}\left(B\left(R_{0}\right)\right)}} \rightarrow \infty$ ). Then $J$ takes a minimum $\varphi_{0}$ (see, e.g., Brezis [1983], Corollary III.20). Moreover if $(\xi, \zeta)$ is the solution of the problem (3.1)-(3.4) with initial datum $\left(\xi_{0}, 0\right)$. We have

$$
\begin{aligned}
& \int_{0}^{T} \int_{\omega_{0}}|\psi|^{p^{\prime}-2} \psi \zeta d x d t-\int_{B(R(T))} \sigma^{d} \xi_{0} d x+ \\
& \epsilon\left\|\varphi^{0}\right\|_{L^{p^{\prime}}(B(R(T)))}^{1-p^{\prime}} \int_{B(R(T))}\left|\varphi^{0}\right|^{p^{\prime}-2} \varphi^{0} \xi^{0} d x=0 .
\end{aligned}
$$

Multiplying (1.1), (1.2) by $(\xi, \zeta)$, integrating by parts and applying Leibnitz Theorem, we arrive to

$$
\begin{gathered}
-\int_{0}^{T}<\sigma, \frac{\partial \xi}{\partial t}>d t-d \int_{0}^{T}<\sigma, \Delta \xi>d t+\int_{0}^{T} \int_{B(R(t))} r_{1} \sigma \xi d x d t+ \\
\int_{0}^{T} \int_{B(R(t))} \lambda \beta \xi d x d t-\int_{0}^{T}<\beta, \frac{\partial \zeta}{\partial t}>d t-d \int_{0}^{T}<\beta, \Delta \zeta>d t+ \\
\left.\left.\int_{0}^{T} \int_{B(R(t))} r_{2} \beta \zeta d x d t-\int_{0}^{T} \int_{\omega_{0}} f \zeta d x d t+\int_{B(R(t))} \sigma \xi d x\right]_{0}^{T}+\int_{B(R(t))} \beta \zeta d x\right]_{0}^{T}=0,
\end{gathered}
$$


where $<,>$ represents the duality $W_{0}^{1, p^{\prime}}\left(B(R(t)) \times W_{0}^{-1, p^{\prime}}(B(R(t))\right.$. From the choice of $(\xi, \zeta)$ and since $\sigma(0, x)=\beta(0, x)=0$ we obtain

$$
-\int_{0}^{T} \int_{\omega_{0}} f \zeta d x d t+\int_{B(R(T))} \sigma(T) \xi^{0} d x=0 .
$$

Now, let us take $f$,

$$
f:=|\psi|^{p^{\prime}-2} \psi .
$$

Substituting it in (3.6) and using (3.5) it results

$$
\int_{B(R(T))}\left(\sigma(T)-\sigma^{d}\right) \xi^{0} d x+\epsilon\left\|\varphi^{0}\right\|_{L^{p^{\prime}}(B(R(T)))}^{1-p^{\prime}} \int_{B(R(T))}\left|\varphi^{0}\right|^{p^{\prime}-2} \varphi^{0} \xi_{0} d x=0
$$

for all $\xi^{0} \in L^{p^{\prime}}(B(R(T)))$. Taking

$$
\xi^{0}=\left(\sigma(T)-\sigma^{d}\right)^{\frac{1}{p^{\prime}-1}} \in L^{p^{\prime}}(B(R(T)))
$$

since $p=1+\frac{1}{p^{\prime}-1}$, we obtain

$$
\begin{gathered}
\| \sigma(T)-\left.\sigma^{d}\right|_{L^{p}(B(R(T)))} ^{p}= \\
\epsilon\left\|\varphi^{0}\right\|_{L^{p^{\prime}}(B(R(T)))}^{1-p^{\prime}} \int_{B(R(T))}\left|\varphi^{0}\right|^{p^{\prime}-2} \varphi^{0}\left|\sigma(T)-\sigma^{d}\right|^{\frac{1}{p^{\prime}-1}-1}\left(\sigma(T)-\sigma^{d}\right) d x .
\end{gathered}
$$

Applying Hölder inequality, we obtain that

$\left\|\varphi^{0}\right\|_{L^{p^{\prime}}(B(R(T)))}^{1-p^{\prime}} \int_{B(R(T))}\left|\varphi^{0}\right|^{p^{\prime}-2} \varphi^{0}\left|\sigma(T)-\sigma^{d}\right|^{\frac{1}{p^{\prime}-1}-1}\left(\sigma(T)-\sigma^{d}\right) d x \leq\left\|\sigma(T)-\sigma^{d}\right\|_{L^{p}(B(R(T)))}^{p-1}$,

which leads to

$$
\left\|\sigma(T)-\sigma^{d}\right\|_{L^{p}(B(R(T)))} \leq \epsilon
$$

and the conclusion holds.

So, it only remains to check the mentioned properties of $J$ :

$\mathbf{J}$ is convex. We express $J$ as addition of the functionals,

$$
\begin{gathered}
J_{1}\left(\varphi^{0}\right):=-\int_{B(R(T))} \sigma^{d} \varphi^{0} d x, \quad J_{2}\left(\varphi^{0}\right):=\epsilon\left\|\varphi^{0}\right\|_{L^{p^{\prime}(B(R(T)))},} \\
J_{3}\left(\varphi^{0}\right):=\frac{1}{p^{\prime}} \int_{0}^{T} \int_{B(R(t))}|\psi|^{p^{\prime}} d x d t .
\end{gathered}
$$

First we shall see that $J_{3}$ is convex. Let $\varphi_{1}^{0}, \varphi_{2}^{0} \in L^{p}(B(R(T)))$ and $\left(\varphi_{1}, \psi_{1}\right)$ and $\left(\varphi_{2}, \psi_{2}\right)$ be the respective solutions of problem $(3.1)-(3.4)$, and let $\alpha \in(0,1)$. Then, since the system is linear we get

$$
J_{3}\left(\alpha \varphi_{1}^{0}+(1-\alpha) \varphi_{2}^{0}\right)=\frac{1}{p^{\prime}} \int_{0}^{T} \int_{B(R(t))}\left(\left|\alpha \psi_{1}+(1-\alpha) \psi_{2}\right|^{p^{\prime}} d x d t\right.
$$

and then

$$
J_{3}\left(\alpha \varphi_{1}^{0}+(1-\alpha) \varphi_{2}^{0}\right)-\alpha J_{3}\left(\varphi_{1}^{0}\right)-(1-\alpha) J_{3}\left(\varphi_{2}^{0}\right)=
$$




$$
=\frac{1}{p^{\prime}} \int_{0}^{T} \int_{B(R(t))}\left(\left|\alpha \psi_{1}+(1-\alpha) \psi_{2}\right|^{p^{\prime}}-\alpha\left|\psi_{1}\right|^{p^{\prime}}-(1-\alpha)\left|\psi_{2}\right|^{p^{\prime}}\right) d x d t .
$$

Since $p^{\prime}>1$ we obtain

$$
\left|\alpha \psi_{1}+(1-\alpha) \psi_{2}\right|^{p^{\prime}}-\alpha\left|\psi_{1}\right|^{p^{\prime}}-(1-\alpha)\left|\psi_{2}\right|^{p^{\prime}} \leq 0
$$

and integrating we obtain,

$$
\frac{1}{p^{\prime}} \int_{0}^{T} \int_{B(R(t))}\left(\left|\alpha \psi_{1}+(1-\alpha) \psi_{2}\right|^{p^{\prime}}-\alpha\left|\psi_{1}\right|^{p^{\prime}}-(1-\alpha)\left|\psi_{2}\right|^{p^{\prime}}\right) d x d t \leq 0,
$$

which proves the convexity of $J_{3}$. Finally $J_{1}$ is linear and so convex and since $\|$. $\|_{L^{p^{\prime}}(B(R(T))}$, is convex, $J_{2}$ is also convex.

$\mathbf{J}$ is continuous. By construction, $J_{1}$ and $J_{2}$ are continuous. Now we shall prove that $J_{3}$ is continuous too. Let $\varphi_{n}^{0} \in L^{p^{\prime}}(B(R(T)))$ such that $\varphi_{n}^{0} \longrightarrow \varphi^{0}$ and let $\left(\varphi_{n}, \psi_{n}\right),(\varphi, \psi)$ be the solutions of the problem (3.1)-(3.4) with initial data $\varphi_{n}^{0}$ and $\varphi^{0}$, respectively. Subtracting both systems and taking

$$
\left(p^{\prime}\left|\varphi-\varphi_{n}\right|^{p^{\prime}-2}\left(\varphi-\varphi_{n}\right), p^{\prime}\left|\psi-\psi_{n}\right|^{p^{\prime}-2}\left(\psi-\psi_{n}\right)\right),
$$

as test function and using the integration by parts formula (see e.g. Alt and Luckhaus [1983]) and Young inequality, we arrive to

$$
\begin{gathered}
-\frac{\partial}{\partial t} \int_{B(R(t))}\left[\left(\varphi-\varphi_{n}\right)^{p^{\prime}}+\left(\psi-\psi_{n}\right)^{p^{\prime}}\right] d x+ \\
\int_{B(R(t))}\left(r_{1} p^{\prime}-|\lambda|\right)\left|\varphi-\varphi_{n}\right|^{p^{\prime}} d x+\int_{B(R(t))}\left(r_{2} p^{\prime}-|\lambda|\right)\left|\psi-\psi_{n}\right|^{p^{\prime}} d x \leq 0 .
\end{gathered}
$$

Denoting by

$$
X_{n}(t)=\left\|\varphi-\varphi_{n}\right\|_{L^{p^{\prime}}(B(R(t)))}^{p^{\prime}}+\left\|\psi-\psi_{n}\right\|_{L^{p^{\prime}}(B(R(t)))}^{p^{\prime}},
$$

we obtain the differential inequality

$$
\begin{aligned}
& -X_{n}^{\prime}(t) \leq C X_{n}(t), \quad t \in(0, T), \\
& X_{n}(T)=\left\|\varphi_{n}^{0}-\varphi^{0}\right\|_{L^{p^{\prime}}(B(R(T))}^{p^{\prime}},
\end{aligned}
$$

where

$$
C=\max \left\{-r_{1} p^{\prime}+|\lambda|,-r_{2} p^{\prime}+|\lambda|\right\}
$$

Thus we obtain

$$
0 \leq X_{n}(t) \leq\left|X_{n}(T)\right| e^{-C(t-T)}
$$

But

$$
0 \leq \int_{\omega_{0}}\left|\psi-\psi_{n}\right|^{p^{\prime}} d x \leq X_{n}(t),
$$

integrating on $[0, T]$ and taking limits as $n \rightarrow \infty$ we conclude that

$$
\int_{0}^{T} \int_{\omega_{0}}\left|\psi-\psi_{n}\right|^{p^{\prime}} d x d t \leq \int_{0}^{T} X_{n}(t) d t \longrightarrow 0,
$$


which shows the continuity of $J_{3}$.

$\mathbf{J}$ is coercive. Let $\varphi_{n}^{0} \in L^{p^{\prime}}(B(R(T)))$ such that $\left\|\varphi_{n}^{0}\right\|_{L^{p^{\prime}}(B(R(T)))} \longrightarrow \infty$, when $n \longrightarrow$ $\infty$. Now, we shall see

$$
\liminf _{n \rightarrow \infty} \frac{J\left(\varphi_{n}^{0}\right)}{\left\|\varphi_{n}^{0}\right\|_{L^{p^{\prime}}(B(R(T)))}} \geq \epsilon .
$$

Let

$$
I:=\liminf _{n \rightarrow \infty} \frac{J\left(\varphi_{n}^{0}\right)}{\left\|\varphi_{n}^{0}\right\|_{L^{p^{\prime}}(B(R(T)))}} \geq-\left\|\sigma^{d}\right\|_{L^{p}(B(R(T)))}
$$

Then there exists a minimizing subsequence, (which we denote again by $\varphi_{n}^{0}$ ) such that

$$
\lim _{n \rightarrow \infty} \frac{J\left(\varphi_{n}^{0}\right)}{\left\|\varphi_{n}^{0}\right\|_{L^{p^{\prime}}(B(R(T)))}}=I .
$$

We define

$$
\bar{\varphi}_{n}^{0}:=\frac{\varphi_{n}^{0}}{\left\|\varphi_{n}^{0}\right\|_{L^{p^{\prime}}(B(R(T)))}}
$$

and denote by $\left(\bar{\varphi}_{n}, \bar{\psi}_{n}\right)$ the solution of problem $(3.1)-(3.4)$ with initial data $\left(\bar{\varphi}_{n}^{0}, 0\right)$. Since the system is linear we have

$$
\left(\bar{\varphi}_{n}, \bar{\psi}_{n}\right)=\frac{1}{\left\|\varphi_{n}^{0}\right\|_{L^{p^{\prime}}}}\left(\varphi_{n}, \psi_{n}\right)
$$

Then

$$
\frac{J\left(\varphi_{n}^{0}\right)}{\left\|\varphi_{n}^{0}\right\|_{L^{p^{\prime}}(B(R(T)))}}=\left\|\varphi_{n}^{0}\right\|^{p^{\prime}-1} \int_{0}^{T} \int_{\omega_{0}} \bar{\psi}_{n}^{p^{\prime}} d x d t-\int_{B(R(T)} \sigma^{d} \bar{\varphi}_{n}^{0} d x+\epsilon .
$$

Now, it is clear that if

$$
\liminf _{n \rightarrow \infty} \int_{0}^{T} \int_{\omega_{0}} \bar{\psi}_{n}^{p^{\prime}} d x \geq \alpha_{0}
$$

for some $\alpha_{0}$ then

$$
\frac{J\left(\varphi_{n}^{0}\right)}{\left\|\varphi_{n}^{0}\right\|_{L^{p^{\prime}}(B(R(T)))}} \geq \alpha_{0}\left\|\varphi_{n}^{0}\right\|_{L^{p^{\prime}(B(R(T)))}}^{p^{\prime}-1}+\epsilon-\left\|\sigma^{d}\right\|_{L^{p}(B(R((T)))} \longrightarrow \infty
$$

as $n \rightarrow \infty$, which proves the property. Let us assume now that lim inf $\int_{0}^{T} \int_{\omega_{0}} \bar{\psi}_{n}^{p^{\prime}} d x=0$. Then there exists a subsequence $\bar{\psi}_{n_{i}}$ such that

$$
\int_{0}^{T} \int_{\omega_{0}}\left|\bar{\psi}_{n_{i}}\right|^{p^{\prime}} d x d t \longrightarrow 0
$$

therefore $\bar{\psi}_{n_{i}} \rightarrow 0$ in $L^{p^{\prime}}\left(\omega_{0} \times[0, T]\right)$. Taking $(0, \zeta)$ as test function in (3.2), where $\zeta \in C_{c}^{2}\left((0, T) \times \omega_{0}\right)$, we obtain

$$
\begin{gathered}
\int_{0}^{T} \int_{\omega_{0}} \bar{\psi}_{n_{i}} \frac{\partial \zeta}{\partial t} d x d t-\int_{0}^{T} \int_{\omega_{0}} \bar{\psi}_{n_{i}} \Delta \zeta d x d t- \\
r_{2} \int_{0}^{T} \int_{\omega_{0}} \bar{\psi}_{n_{i}} \zeta d x d t+\lambda \int_{0}^{T} \int_{\omega_{0}} \bar{\varphi}_{n_{i}} \zeta d x d t=0 .
\end{gathered}
$$


Now, passing to the limit when $n_{i} \longrightarrow \infty$ it results

$$
\int_{0}^{T} \int_{\omega_{0}} \bar{\varphi}_{n_{i}} \zeta d x d t \longrightarrow 0
$$

where $\bar{\varphi}_{n_{i}}$ is the solution of the problem

$$
\left\{\begin{array}{l}
-\frac{\partial \bar{\varphi}_{n_{i}}}{\partial t}-D_{1} \Delta \bar{\varphi}_{n_{i}}-r_{1} \bar{\varphi}_{n_{i}}=0, \quad|x|<R(t), t \in(0, T), \\
\bar{\varphi}_{n_{i}}(0, x)=\bar{\varphi}^{0}
\end{array}\right.
$$

Making the change of variable (2.1), and

$$
\bar{u}_{n_{i}}(\tilde{x}, \tilde{t}):=\bar{\varphi}_{n_{i}}(R(t(\tilde{t})) \tilde{x}, t(\tilde{t})),
$$

we obtain

$$
\begin{cases}-\frac{\partial \bar{u}_{n_{i}}}{\partial \tilde{t}}-D \Delta \bar{u}_{n_{i}}-R^{2} R^{\prime} \tilde{x} \cdot \nabla \bar{u}_{n_{i}}+R^{2} r_{1} \bar{u}_{n_{i}}=0, & |\tilde{x}|<1, \tilde{t} \in(0, \tilde{T}), \\ \bar{u}_{n_{i}}(\tilde{x}, \tilde{t})=0, & |\tilde{x}|=1, \tilde{t} \in(0, \tilde{T}), \\ \bar{u}_{n_{i}}(\tilde{x}, 0)=u_{0}(\tilde{x})=\bar{\varphi}_{n_{i}}^{0}\left(\tilde{x} R_{0}\right), & |\tilde{x}|<1,\end{cases}
$$

such that $\bar{u}_{n_{i}}^{0} \rightarrow \bar{u}_{0}$ in $L^{p^{\prime}}(B)$, and furthermore $\bar{u}_{n_{i}} \rightarrow \bar{u}$ solution of (3.10), with initial data $\bar{u}_{0}=\bar{\varphi}_{n_{i}}^{0}$. By $(3.8), \bar{u}_{n_{i}} \longrightarrow 0$, weakly in $L^{p^{\prime}}\left(B\left(\hat{\omega}_{0}\right)\right)$, where $\hat{\omega}_{0}$ is an open subset of $B$, such that $\hat{\omega}_{0} \subset \tilde{\omega}_{0}$. Consequently $\bar{u} \equiv 0$ on $\tilde{\omega}_{0}$ for all $0 \leq \tilde{t} \leq \tilde{T}$. By the unique continuation for the equation (3.10) (see Chi-Cheung Poon [1996], Theorem 1.1') we deduce that $\bar{u}=0$ in $B \times(0, \tilde{T})$, and by the uniqueness of problem (3.10), it result $\bar{u}_{0} \equiv 0$ and $\bar{\varphi}^{0} \equiv 0$. Furthermore

$$
-\int_{B(R(T))} \sigma^{d} \bar{\varphi}^{0} d x=0
$$

and $I=\epsilon$, from where we deduce that $J$ is coercive.

\section{Proof of the Theorem 1.1.}

We construct the sequence $\left\{R_{n}(t)\right\}$, such that $R_{n}$ verifies

$$
R_{n}^{2}(t) \dot{R}_{n}(t)=\int_{B\left(R_{n-1}(t)\right)} S\left(\sigma_{n-1}+\sigma_{n-1}^{s}, \beta_{n-1}+\beta_{n-1}^{s}\right) d x, \quad R_{n}(0)=R_{0},
$$

for $n>1$, where $\left(\sigma_{n-1}^{s}, \beta_{n-1}^{s}\right)$ is the solution of the problem (1.1), (1.2), (1.4) and (1.5), with $f \equiv 0$, and initial data $\sigma_{n-1}^{s}(x, 0)=\sigma_{0}(x), \beta_{n-1}^{s}(x, 0)=\beta_{0}(x)$, and $R(t)=R_{n-1}(t)$, and $\left(\sigma_{n-1}, \beta_{n-1}\right)$ is the solution mentioned in Proposition 3.1. We start the process by taking, e.g. $R_{1}(t)=R_{0}$. Since $S$ is bounded, $R_{n} \in W^{1, \infty}(0, T)$ and we deduce there exists a subsequence of functions $R_{n_{i}}$ such that converges weakly to $R(t)$ in $W^{1, q}(0, T)$, for all $q \in(1, \infty)$. By Proposition 3.1, for each $R_{n}$ there exists a minimum function $\varphi_{n}^{0}$. We shall show that the sequence $\left\|\varphi_{n}^{0}\right\|_{L^{p^{\prime}}(B(R(T)))}$ is uniformly bounded. We consider

$$
J_{n}\left(\varphi_{n}^{0}\right):=\int_{0}^{T} \int_{\omega_{0}}\left|\psi_{n}\right|^{p^{\prime}} d x d t+\epsilon\left\|\varphi_{n}^{0}\right\|_{L^{p^{\prime}}\left(B\left(R_{n}(T)\right)\right)}-\int_{B\left(R_{n}(T)\right)} \sigma_{n}^{d} \varphi_{0}^{n} d x
$$


where $\sigma_{n}^{d}=\hat{\sigma}^{d} \chi_{B\left(R_{n}(T)\right)}$. Supposing $\left\|\varphi_{n}^{0}\right\|_{L^{p^{\prime}}\left(B\left(R_{n}(T)\right)\right)} \longrightarrow \infty$, since $J_{n}(0)=0$ and (by definition of $\left.\varphi_{n}^{0}\right), J_{n}\left(\varphi_{n}^{0}\right) \leq 0$,

$$
\frac{J_{n}\left(\varphi_{n}^{0}\right)}{\left\|\varphi_{n}^{0}\right\|_{L^{p^{\prime}}}}=\left\|\varphi_{n}^{0}\right\|_{L^{\prime}\left(B\left(R_{n}(T)\right)\right)}^{p^{\prime}-1} \int_{0}^{T} \int_{\omega_{0}} \bar{\psi}_{n}^{p} d x d t+\epsilon-\int_{B\left(R_{n}(T)\right)} \sigma_{n}^{d} \bar{\varphi}_{n}^{0} d x \leq 0,
$$

since

$$
\int_{B\left(R_{n}(T)\right)} \sigma_{n}^{d} \frac{\varphi_{n}^{0}}{\left\|\varphi_{n}^{0}\right\|_{L^{p^{\prime}}(B(R(T)))}} d x \leq\left\|\sigma_{n}^{d}\right\|_{L^{p}\left(B\left(R_{n}(T)\right)\right)} \leq\left\|\hat{\sigma}^{d}\right\|_{L^{p}\left(B\left(R_{0} \exp \left\{\|S\|_{L^{\infty}} \infty\right\}\right)\right)},
$$

it results, by (3.11),

$$
\int_{0}^{T} \int_{\omega_{0}} \bar{\psi}_{n}^{p^{\prime}} d x d t \longrightarrow 0 \quad \text { when } \quad n \longrightarrow \infty
$$

Repeating the argument used in the proof that $J$ is coercive, we obtain

$$
\bar{\varphi}_{0}^{n} \rightarrow 0 \text { in } L^{p^{\prime}}(B(R(T)))
$$

and

$$
\liminf _{n \rightarrow \infty} \frac{J_{n}\left(\varphi_{n}^{0}\right)}{\left\|\varphi_{n}^{0}\right\|} \geq \epsilon
$$

which is a contradiction with (3.11). Consequently $\left\|\varphi_{n}^{0}\right\|_{L^{p^{\prime}\left(B\left(R_{n}(T)\right)\right)}}$ is uniformly bounded

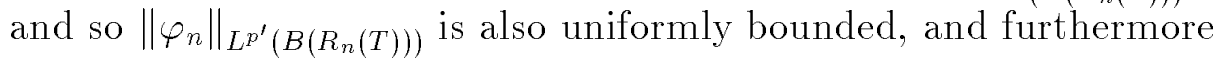

$$
\left\|f_{n}\right\|_{L^{p}\left(0, T: L^{p}\left(\omega_{0}\right)\right)} \leq C,
$$

for some $C$ independent of $n$.

Making the change of variable (2.1), (2.2), by Lemma 2.1, we obtain that if $\left(u_{n}, v_{n}, R_{n}\right)$ is the transformed of $\left(\sigma_{n}+\sigma_{n}^{s}, \beta_{n}+\beta_{n}^{s}, R_{n}\right)$ then it is uniformly bounded in ( $W^{1, p}(B \times$ $\left.(0, \tilde{T}))^{2}, H^{2}(0, T)\right)$, and by compact embedding, there exists a subsequence $\left(u_{n i}, v_{n i}, R_{n i}\right)$ such that converges strongly in $\left(C^{\alpha}((0, T] \times B)^{2}, C^{1}([0, T])\right)$, to $(u, v, R)$ for $\alpha=\frac{1}{6}$, where $\left(u_{n i}, v_{n i}\right)$ satisfies

$$
\begin{cases}\frac{\partial u_{n i}}{\partial t}-\frac{d}{R_{n i}^{2}} \Delta u_{n i}-\frac{R_{n i}^{\prime}}{R_{n i}} \tilde{x} \cdot \nabla u_{n i}+r_{1} u_{n i}+\lambda v_{n i}=0, & |\tilde{x}|<1, t \in(0, T), \\ \frac{\partial v_{n i}}{\partial t}-\frac{d}{R_{n i}^{2}} \Delta v_{n i}-\frac{R_{n i}^{\prime}}{R_{n i}} \tilde{x} \cdot \nabla v_{n i}+r_{2} v_{n i}=f_{n} \chi_{\tilde{\omega}_{0}}, & |\tilde{x}|<1, t \in(0, T), \\ u_{n i}(\tilde{x}, t)=v_{n i}(\tilde{x}, t)=0, & |\tilde{x}|=1, t \in(0, T), \\ u_{n i}(\tilde{x}, 0)=u_{n i}^{0}(\tilde{x}), v_{n i}(\tilde{x}, 0)=v_{n i}^{0}(\tilde{x}), & |\tilde{x}|<1,\end{cases}
$$

and $(u, v, R)$ is solution of $(2.3)-(2.7)$. In particular

$$
\left\|u(T)-u_{n}(T)\right\|_{L^{p}(B)}^{p} \longrightarrow 0, \quad \text { as } n_{i} \rightarrow+\infty .
$$

Moreover

$$
\left\|\sigma(T)-\sigma^{d}\right\|_{L^{p}(B(R(T)))}=\left\|\sigma(T)-\sigma_{n}(T)\right\|_{L^{p}\left(B\left(\min \left\{R(T), R_{n}(T)\right\}\right)\right)}+
$$




$$
\left\|\sigma_{n}(T)-\sigma^{d}\right\|_{L^{p}\left(B\left(\min \left\{R(T), R_{n}(T)\right\}\right)\right)}+\left\|\sigma-\sigma^{d}\right\|_{L^{p}\left(B_{n}^{*}(T)\right)}
$$

where

$$
B_{n}^{*}(T)= \begin{cases}B(R(T)) \cap B^{c}\left(B\left(R_{n}(T)\right)\right), & \text { if } R(T)>R_{n}(T), \\ \emptyset, & \text { if } R(T) \leq R_{n}(T) .\end{cases}
$$

Making the change of variable (2.1), and since

$$
\left\|\sigma_{n}(T)-\sigma^{d}\right\|_{L^{p}\left(B\left(\min \left\{R(T), R_{n}(T)\right\}\right)\right)} \leq \epsilon,
$$

we obtain

$$
\left\|\sigma(T)-\sigma^{d}\right\|_{L^{p}(B(R(T)))} \leq\left\|u(T)-u_{n}(T)\right\|_{L^{p}(B)}+\left\|\sigma-\sigma^{d}\right\|_{L^{p}\left(B_{n}^{*}(T)\right)}+\epsilon .
$$

Since $\left|\sigma-\sigma^{d}\right|^{p} \chi_{B_{n}^{*}(T)} \leq\left|\sigma-\sigma^{d}\right|^{p}$ and $\mu\left(B_{n}^{*}(T)\right) \longrightarrow 0$, by the Lebesgue dominated convergence theorem we obtain that

$$
\lim _{n \rightarrow \infty}\left\|\sigma-\sigma^{d}\right\|_{L^{p}\left(B_{n}^{*}(T)\right)}=0 .
$$

Taking limits when $n \longrightarrow \infty$ it results

$$
\left\|\sigma(T)-\sigma^{d}\right\|_{L^{p}(B(R(T)))} \leq \epsilon,
$$

and the theorem is thereby proved in the case $p \geq 5$.

In the case $p<5$, we consider the control $f$ for $p=5$, then

$$
\left\|\sigma(T)-\sigma^{d}\right\|_{L^{p}(B(R(T)))} \leq \frac{3 \pi}{4} B(R(T))\left\|\sigma(T)-\sigma^{d}\right\|_{L^{5}(B(R(T)))} \leq \frac{3 \pi}{4} \exp \left\{T\|S\|_{L^{\infty}}\right\} \epsilon,
$$

taking $\epsilon=\epsilon^{\prime}\left(\frac{3 \pi}{4} \exp \left\{T\|S\|_{L^{\infty}}\right\}\right)^{-1}$ we conclude the Theorem.

Remark 3.1 Notice that the final observation is made on the density $\sigma(T, \cdot)$ and that once we chose the control in order to have (1.6) the free boundary, $R(t)$, and the inhibitor density $\beta(T, \cdot)$ are univocally determined.

\section{References}

[1] J.A. ADAM, "A simplified mathematical model of tumor growth", Mathematical Biosciences, 81, (1986), pp. 229-244.

[2] H.W. Alt And S. Luckhaus, "Quasi-linear elliptic-parabolic differential equations", Math. Z., 183, (1983), pp. 311-341.

[3] H. Brezis, Analyse Fonctionnelle. Masson, Paris, 1983.

[4] H.M. Byrne and A.J. Chaplain, "Growth of necrotic tumors in the presence and absence of inhibitors", Mathematical Biosciences. 135, (1996), pp. 187-216.

[5] Chi-Cheung Poon, "Unique continuation for parabolic equations", Commun. in Partial Differential Equations, 21, (1996), pp. 521-539. 
[6] A.J. Chaplain, A.R.A. Anderson, "Mathematical modelling, simulation and prediction of tumour-induced Angiogenesis", Invasion and Metastasis, 16, 1996 , pp. 222, 234.

[7] S. Cui, A. Friedman, "Analysis of a mathematical model of effect of inhibitors on the growth of tumors", Journal of Math. Analysis and Applications 236, (1999), pp. 171-206.

[8] S. Cui, A. Friedman, "Analysis of a mathematical model of the growth of the necrotic tumors", Mathematical Biosciences, 159, (1999), pp. 123-144.

[9] J.I. Díaz, A.M. Ramos, "Positive and negative approximate controllability results for semilinear parabolic equations". Revista de la Real Academia de Ciencias Exactas, Físicas y Nat. de Madrid, Tomo LXXXIX,11-30, 1995

[10] J.I. Díaz, A.M. Ramos, "On the Approximate Controllability for Higher Order Parabolic Nonlinear Equations of Cahn-Hilliard Type Control and Estimation of Distributed Parameters Systems", International Series of Numerical Mathematics, Vol. 126, 1998, Birkhäuser Verlag, Bassel, 1998, pp. 111-127.

[11] J.I. DíAz, A.M. Ramos, "Numerical experiences regarding the localized control of nonlinear parabolic problems", In the CD-Rom Proceedings of the European Congress on Computational Methods in Applied Sciences and Engineering (ECCOMAS 2000), Barcelona, 11-14 September 2000, E.Oñate et al. editors, Barcelona, ISBN: 84-89925-70-4.

[12] J.I. DíAz, J.I. TEllo, "A mathematical analysis of a model of the growth of necrotic tumors in presence of inhibitors", submitted 2001. (See also I.M.A. Preprints Series \#1727, University of Minnesota, October 2000).

[13] C. Fabre, J.P. Puel, E. Zuazua, "Approximate controllability of the semilinear heat equation", Proc. Roy. Soc. Edinburgh, Sect. A, 125, (1995), pp. 31-61.

[14] A. Friedman, Partial differential equations of parabolic type, Prentice-Hall, Englewood Cliffs, New Jersey, 1964.

[15] A. Friedman, F. Reitich, "Analysis of a mathematical model for the growth of tumors", J. Math. Biology, 38, (1999), pp. 262-284.

[16] H.P. Greenspan, "Models of the growth of a solid tumor diffusion". Studies in Appl. Math., 52, (1972), pp. 317-340.

[17] R. Glowinski, J.L. Lions, "Exact and approximate controllability for distributed parameter systems, Part II", Acta Numerica, (1995), pp. 157-333.

[18] O.H. Ladyzenkaya, V.A. Solonnikov, N.N. Uralceva Linear and quasilinear equations of parabolic type, Translations of Mathematical Monographs, 23, Amer. Math. Soc., Providence, 1991. 
[19] J.L. Lions, "Remarques sur la contrôllabilité approchée", Actas de Jornadas Hispano-Francesas sobre Control de Sistemas Distribuidos, Univ. de Malaga, 1990, pp. $77-88$.

[20] J.L. Lions, "Exact Controllability for distributed systems: Some trends ans some problems", Applied and Industrial Mathematics, 1991, pp. 59-84.

[21] J. Simon, "Compact sets in the space $L^{p}((0, T), B)$ ", J. Annali Mat. Pura Appl. 146, (1987), pp. 65-96.

[22] J.I. Tello, Tratamiento matemático de procesos físicos con difusión y convección. Ph.D. Thesis, Universidad Complutense de Madrid, Madrid 2001. 